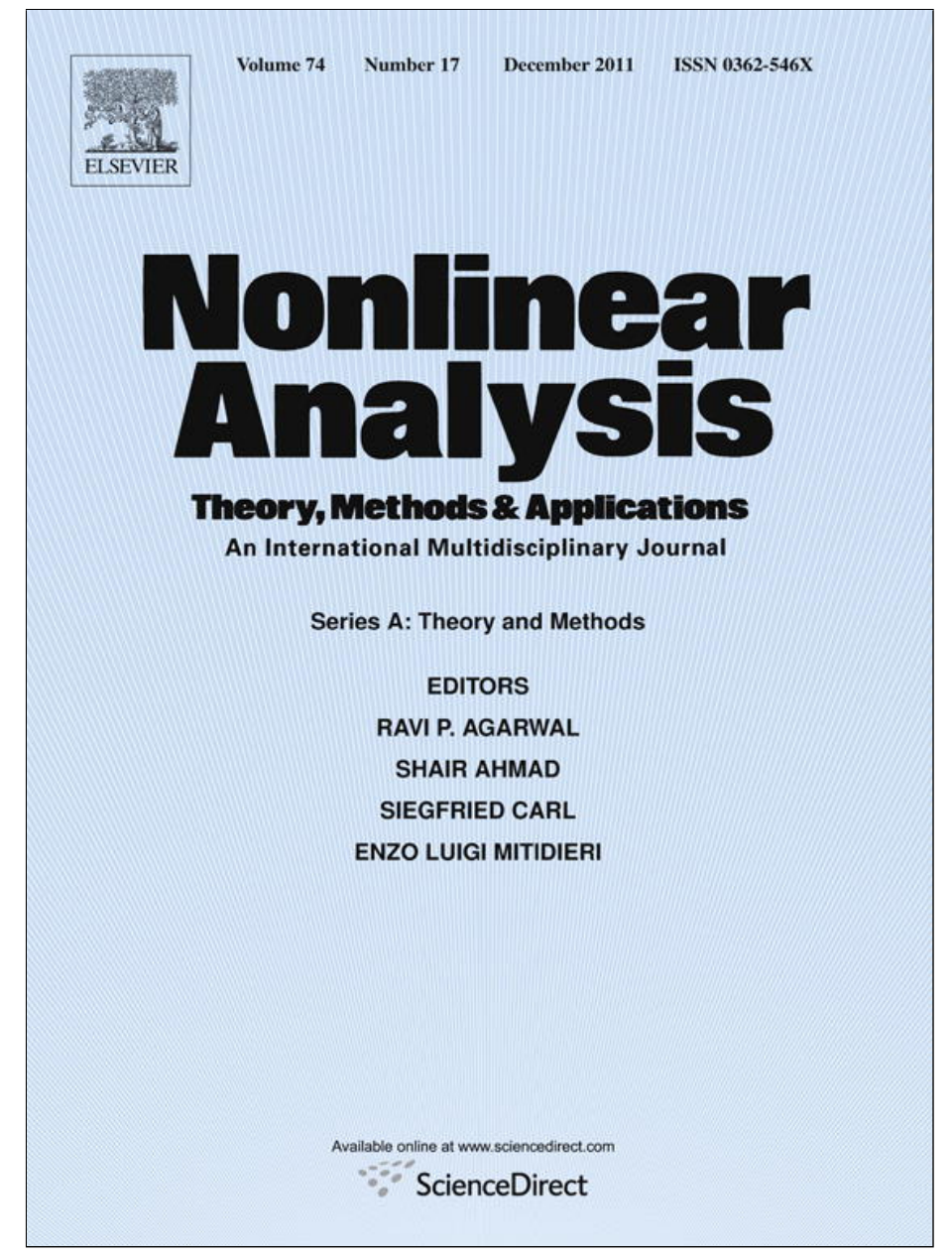

This article appeared in a journal published by Elsevier. The attached copy is furnished to the author for internal non-commercial research and education use, including for instruction at the authors institution and sharing with colleagues.

Other uses, including reproduction and distribution, or selling or licensing copies, or posting to personal, institutional or third party websites are prohibited.

In most cases authors are permitted to post their version of the article (e.g. in Word or Tex form) to their personal website or institutional repository. Authors requiring further information regarding Elsevier's archiving and manuscript policies are encouraged to visit:

http://www.elsevier.com/copyright 


\title{
Competitive Lotka-Volterra population dynamics with jumps
}

\author{
Jianhai Bao ${ }^{\mathrm{a}}$, Xuerong Mao ${ }^{\mathrm{b}}$, Geroge Yin ${ }^{\mathrm{c}}$, Chenggui Yuan ${ }^{\mathrm{a}, *}$ \\ a Department of Mathematics, Swansea University, Swansea SA2 8PP, UK \\ ${ }^{\mathrm{b}}$ Department of Statistics and Modelling Science, University of Strathclyde, Glasgow G1 1XH, UK

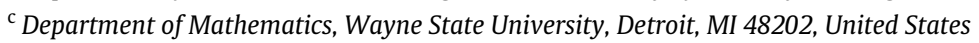

\section{A R T I C L E I N F O}

\section{Article history:}

Received 9 February 2011

Accepted 25 June 2011

Communicated by S. Carl

\section{MSC:}

93D05

$60 \mathrm{~J} 60$

$60 \mathrm{~J} 05$

Keywords:

Lotka-Volterra model

Jumps

Stochastic boundedness

Lyapunov exponent

Variation-of-constants formula

Extinction

\begin{abstract}
A B S T R A C T
This paper considers competitive Lotka-Volterra population dynamics with jumps. The contributions of this paper are as follows. (a) We show that a stochastic differential equation (SDE) with jumps associated with the model has a unique global positive solution; (b) we discuss the uniform boundedness of the pth moment with $p>0$ and reveal the sample Lyapunov exponents; (c) using a variation-of-constants formula for a class of SDEs with jumps, we provide an explicit solution for one-dimensional competitive Lotka-Volterra population dynamics with jumps, and investigate the sample Lyapunov exponent for each component and the extinction of our $n$-dimensional model.
\end{abstract}

(c) 2011 Elsevier Ltd. All rights reserved.

\section{Introduction}

The differential equation

$$
\left\{\begin{array}{l}
\frac{d X(t)}{d t}=X(t)[a(t)-b(t) X(t)], \quad t \geq 0, \\
X(0)=x,
\end{array}\right.
$$

has been used to model the population growth of a single species whose members usually live in proximity, share the same basic requirements, and compete for resources, food, habitat, or territory, and is known as the competitive Lotka-Volterra model or logistic equation. The competitive Lotka-Volterra model for $n$ interacting species is described by the $n$-dimensional differential equation

$$
\frac{d X_{i}(t)}{d t}=X_{i}(t)\left[a_{i}(t)-\sum_{j=1}^{n} b_{i j}(t) X_{j}(t)\right], \quad i=1,2, \ldots, n,
$$

where $X_{i}(t)$ represents the population size of species $i$ at time $t, a_{i}(t)$ is the rate of growth at time $t, b_{i j}(t)$ represents the effect of interspecies (if $i \neq j$ ) or intraspecies (if $i=j$ ) interaction at time $t$, and $a_{i}(t) / b_{i j}(t)$ is the carrying capacity of the $i$ th

\footnotetext{
* Corresponding author. Tel.: +44 1792 602228; fax: +44 1792295843.

E-mail addresses: jianhaibao@yahoo.com.cn (J. Bao), xuerong@stams.strath.ac.uk (X. Mao), gyin@math.wayne.edu (G. Yin), C.Yuan@swansea.ac.uk (C. Yuan).
} 
species in the absence of other species at time $t$. Eq. (1.1) takes the matrix form

$$
\frac{d X(t)}{d t}=\operatorname{diag}\left(X_{1}(t), \ldots, X_{n}(t)\right)[a(t)-B(t) X(t)],
$$

where

$$
X=\left(X_{1}, \ldots, X_{n}\right)^{T}, \quad a=\left(a_{1}, \ldots, a_{n}\right)^{T}, \quad B=\left(b_{i j}\right)_{n \times n} .
$$

There is an extensive literature concerned with the dynamics of Eq. (1.2) and here we only mention Gopalsamy [1], Kuang [2], Li et al. [3], Takeuchi and Adachi [4,5], and Xiao and Li [6]. In particular, the books by Gopalsamy [1] and Kuang [2] are good references in this area.

On the other hand, the deterministic models assume that parameters in the systems are all deterministic irrespective of environmental fluctuations, which, from the biological point of view, imposes some limitations in mathematical modelling of ecological systems; population dynamics in the real world is inevitably affected by environmental noise-see, e.g., Gard [7,8]. Therefore, competitive Lotka-Volterra models in random environments are becoming more and more popular. In general, there are two ways considered in the literature for modelling the influence of environmental fluctuations in population dynamics. One is to consider random perturbations of interspecies or intraspecies interactions by white noise. Recently, Mao et al. [9] investigated the stochastic $n$-dimensional Lotka-Volterra system

$$
d X(t)=\operatorname{diag}\left(X_{1}(t), \ldots, X_{n}(t)\right)[(a+B X(t)) d t+\sigma X(t) d W(t)],
$$

where $W$ is a one-dimensional standard Brownian motion, and revealed that the environmental noise can suppress a potential population explosion (see, e.g., [10,11], among others, in this connection). Another is to consider the stochastic perturbation of the growth rate $a(t)$ by a white noise with

$$
a(t) \rightarrow a(t)+\sigma(t) \dot{W}(t),
$$

where $\dot{W}(t)$ is a white noise, namely, $W(t)$ is a Brownian motion defined on a complete probability space $(\Omega, \mathcal{F}, \mathbb{P})$ with a filtration $\{\mathscr{F}\}_{t \geq 0}$ satisfying the usual conditions (i.e., it is right continuous and increasing while $\mathscr{F}_{0}$ contains all $\mathbb{P}$-null sets). As a result, Eq. (1.2) becomes a competitive Lotka-Volterra model in random environments:

$$
d X(t)=\operatorname{diag}\left(X_{1}(t), \ldots, X_{n}(t)\right)[(a(t)-B(t) X(t)) d t+\sigma(t) d W(t)] .
$$

There is also extensive literature concerning all kinds of properties of model (1.4); see, e.g., Hu and Wang [12], Jiang and Shi [13], Liu and Wang [14], Zhu and Yin [15,16], and the references therein.

Furthermore, the population may suffer sudden environmental shocks, e.g., earthquakes, hurricanes, epidemics, etc. However, stochastic Lotka-Volterra model (1.4) cannot explain such phenomena. To explain these phenomena, introducing a jump process into the underlying population dynamics provides a feasible and more realistic model. In this paper, we develop Lotka-Volterra model with jumps:

$$
d X(t)=\operatorname{diag}\left(X_{1}\left(t^{-}\right), \ldots, X_{n}\left(t^{-}\right)\right)\left[\left(a(t)-B(t) X\left(t^{-}\right)\right) d t+\sigma(t) d W(t)+\int_{\mathbb{Y}} \gamma(t, u) \tilde{N}(d t, d u)\right] .
$$

Here $X\left(t^{-}\right)$is the left limit of $X(t), a, B$ are defined as in Eq. (1.2),

$$
\sigma=\left(\sigma_{1}, \ldots, \sigma_{n}\right)^{T}, \quad \gamma=\left(\gamma_{1}, \ldots, \gamma_{n}\right)^{T},
$$

$W$ is a real-valued standard Brownian motion, $N$ is a Poisson counting measure with characteristic measure $\lambda$ on a measurable subset $\mathbb{Y}$ of $[0, \infty)$ with $\lambda(\mathbb{Y})<\infty$, and $\tilde{N}(d t, d u):=N(d t, d u)-\lambda(d u) d t$. Throughout the paper, we assume that $W$ and $N$ are independent.

An example is provided by bee colonies in a field [17]. In particular, they compete for food strongly with the colonies located near to them. Similar phenomena abound in nature; see, e.g., [18]. Hence it is reasonable to assume that the selfregulating competitions within the same species are strictly positive; see, e.g., [15,16]. Therefore we also assume:

(A) For any $t \geq 0$ and $i, j=1,2, \ldots, n$ with $i \neq j, a_{i}(t)>0, b_{i i}(t)>0, b_{i j}(t) \geq 0, \sigma_{i}(t)$ and $\gamma_{i}(t, u)$ are bounded functions, $\hat{b}_{i i}:=\inf _{t \in \mathbb{R}_{+}} b_{i i}(t)>0$ and $\gamma_{i}(t, u)>-1, u \in \mathbb{Y}$.

With reference to the existing results in the literature, our contributions are as follows:

- We use jump diffusion to model the evolutions of population dynamics.

- We demonstrate that if the population dynamics with jumps is self-regulating or competitive, then the population will not explode in a finite time almost surely.

- We discuss the uniform boundedness of the $p$ th moment for any $p>0$ and reveal the sample Lyapunov exponents.

- We obtain the explicit expression for the one-dimensional competitive Lotka-Volterra model with jumps, and the uniqueness of the invariant measure, and further reveal precisely the sample Lyapunov exponents for each component and investigate its extinction. 


\section{Global positive solutions}

As the $i$ th state $X_{i}(t)$ of Eq. (1.5) denotes the size of the $i$ th species in the system, it should be nonnegative. Moreover, in order to guarantee that SDEs have a unique global (i.e., no explosion in a finite time) solution for any given initial data, the coefficients of the equation are generally required to satisfy the linear growth and local Lipschitz conditions; see, e.g., [11]. However, the drift coefficient of Eq. (1.5) does not satisfy the linear growth condition, though it is locally Lipschitz continuous, so the solution of Eq. (1.5) may explode in a finite time. It is therefore necessary to provide some conditions under which the solution of Eq. (1.5) not only is positive but also will not explode to infinity in any finite time.

Throughout this paper, $K$ denotes a generic constant whose values may vary at its different appearances. For a bounded function $v$ defined on $\mathbb{R}_{+}$, set

$$
\hat{v}:=\inf _{t \in \mathbb{R}_{+}} v(t) \quad \text { and } \quad \check{v}:=\sup _{t \in \mathbb{R}_{+}} v(t) \text {. }
$$

For convenience of reference, we recall some fundamental inequalities stated as a lemma.

\section{Lemma 2.1.}

$$
\begin{aligned}
& x^{r} \leq 1+r(x-1), \quad x \geq 0, \quad 1 \geq r \geq 0, \\
& n^{\left(1-\frac{p}{2}\right) \wedge 0}|x|^{p} \leq \sum_{i=1}^{n} x_{i}^{p} \leq n^{\left(1-\frac{p}{2}\right) \vee 0}|x|^{p}, \quad \forall p>0, x \in \mathbb{R}_{+}^{n},
\end{aligned}
$$

where $\mathbb{R}_{+}^{n}:=\left\{x \in \mathbb{R}^{n}: x_{i}>0,1 \leq i \leq n\right\}$, and

$$
\ln x \leq x-1, \quad x>0 \text {. }
$$

Theorem 2.1. Under assumption (A), for any initial condition $X(0)=x_{0} \in \mathbb{R}_{+}^{n}$, Eq. (1.5) has a unique global solution $X(t) \in \mathbb{R}_{+}^{n}$ for any $t \geq 0$ almost surely.

Proof. Since the drift coefficient does not fulfil the linear growth condition, the general theorems of existence and uniqueness cannot be implemented for this equation. However, it is locally Lipschitz continuous; therefore for any given initial condition $X(0) \in \mathbb{R}_{+}^{n}$ there is a unique local solution $X(t)$ for $t \in\left[0, \tau_{e}\right.$ ), where $\tau_{e}$ is the explosion time. By Eq. (1.5) the $i$ th component $X_{i}(t)$ of $X(t)$ admits the form for $i=1, \ldots, n$

$$
d X_{i}(t)=X_{i}\left(t^{-}\right)\left[\left(a_{i}(t)-\sum_{j=1}^{n} b_{i j}(t) X_{j}\left(t^{-}\right)\right) d t+\sigma_{i}(t) d W(t)+\int_{\mathbb{Y}} \gamma_{i}(t, u) \tilde{N}(d t, d u)\right] .
$$

Noting that for any $t \in\left[0, \tau_{e}\right)$

$$
\begin{aligned}
X_{i}(t)= & X_{i}(0) \exp \left\{\int_{0}^{t}\left(a_{i}(s)-\sum_{j=1}^{n} b_{i j}(s) X_{j}(s)-\frac{1}{2} \sigma_{i}^{2}(s)+\int_{\mathbb{Y}}\left(\ln \left(1+\gamma_{i}(s, u)\right)-\gamma_{i}(s, u)\right) \lambda(d u)\right) d s\right. \\
& \left.+\int_{0}^{t} \sigma_{i}(s) d W(s)+\int_{0}^{t} \int_{\mathbb{Y}} \ln \left(1+\gamma_{i}(s, u)\right) \tilde{N}(d s, d u)\right\}
\end{aligned}
$$

together with $X_{i}(0)>0$, we can conclude that $X_{i}(t) \geq 0$ for any $t \in\left[0, \tau_{e}\right)$. Now consider the following two auxiliary SDEs with jumps:

$$
\begin{aligned}
& d Y_{i}(t)=Y_{i}\left(t^{-}\right)\left[\left(a_{i}(t)-b_{i i}(t) Y_{i}\left(t^{-}\right)\right) d t+\sigma_{i}(t) d W(t)+\int_{\mathbb{Y}} \gamma_{i}(t, u) \tilde{N}(d t, d u)\right], \\
& Y_{i}(0)=X_{i}(0)
\end{aligned}
$$

and

$$
\begin{aligned}
& d Z_{i}(t)=Z_{i}\left(t^{-}\right)\left[\left(a_{i}(t)-\sum_{i \neq j} b_{i j}(t) Y_{j}(t)-b_{i i}(t) Z_{i}\left(t^{-}\right)\right) d t+\sigma_{i}(t) d W(t)+\int_{\mathbb{Y}} \gamma_{i}(t, u) \tilde{N}(d t, d u)\right], \\
& Z_{i}(0)=X_{i}(0) .
\end{aligned}
$$

Due to $1+\gamma_{i}(t, u)>0$ by (A), it follows that for any $x_{2} \geq x_{1}$,

$$
\left(1+\gamma_{i}(t, u)\right) x_{2} \geq\left(1+\gamma_{i}(t, u)\right) x_{1} .
$$

Then by the comparison theorem [19, Theorem 3.1] we can conclude that

$$
Z_{i}(t) \leq X_{i}(t) \leq Y_{i}(t), \quad t \in\left[0, \tau_{e}\right) .
$$


By Lemma 4.2 below, for $Y_{i}(0)\left(=X_{i}(0)\right)>0$, we know that $Y_{i}(t)$ will not explode in any finite time. Moreover, like for Lemma 4.2 below for $Z_{i}(0)\left(=X_{i}(0)\right)>0$, we can show that

$$
\mathbb{P}\left(Z_{i}(t)>0 \text { on } t \in\left[0, \tau_{e}\right)\right)=1 .
$$

Hence $\tau_{e}=\infty$ and $X_{i}(t)>0$ almost surely for any $t \in[0, \infty)$. The proof is therefore complete.

\section{Boundedness, tightness, and Lyapunov-type exponents}

In the previous section, we saw that Eq. (1.5) has a unique global solution $X(t) \in \mathbb{R}_{+}^{n}$ for any $t \geq 0$ almost surely. In this part we shall show for any $p>0$ the solution $X(t)$ of Eq. (1.5) admits a uniformly finite $p$ th moment, and discuss the long-term behaviours.

Theorem 3.1. Let assumption (A) hold.

(1) For any $p \in[0,1$,$] there is a constant K$ such that

$$
\sup _{t \in \mathbb{R}_{+}} \mathbb{E}|X(t)|^{p} \leq K .
$$

(2) Assume further that there exists a constant $\bar{K}(p)>0$ such that for some $p>1, t \geq 0, i=1, \ldots, n$,

$$
\int_{\mathbb{Y}}\left|\gamma_{i}(t, u)\right|^{p} \lambda(d u) \leq \bar{K}(p) .
$$

Then there exists a constant $K(p)>0$ such that

$$
\sup _{t \in \mathbb{R}_{+}} \mathbb{E}|X(t)|^{p} \leq K(p) .
$$

Proof. We shall prove (3.3) first. Define a Lyapunov function for $p>1$ :

$$
V(x):=\sum_{i=1}^{n} x_{i}^{p}, \quad x \in \mathbb{R}_{+}^{n} .
$$

Applying the Itô formula, we obtain

$$
\mathbb{E}\left(e^{t} V(X(t))\right)=V\left(x_{0}\right)+\mathbb{E} \int_{0}^{t} e^{s}[V(X(s))+\mathscr{L} V(X(s), s)] d s,
$$

where, for $x \in \mathbb{R}_{+}^{n}$ and $t \geq 0$,

$$
\mathcal{L} V(x, t):=p \sum_{i=1}^{n}\left[a_{i}(t)-\sum_{j=1}^{n} b_{i j}(t) x_{j}-\frac{(1-p) \sigma_{i}^{2}(t)}{2}\right] x_{i}^{p}+\sum_{i=1}^{n} \int_{\mathbb{Y}}\left[\left(1+\gamma_{i}(t, u)\right)^{p}-1-p \gamma_{i}(t, u)\right] \lambda(d u) x_{i}^{p} .
$$

By assumption (A) and (3.2), we can deduce that there exists a constant $K>0$ such that

$$
\begin{aligned}
V(x)+\mathscr{L} V(x, t) \leq & \sum_{i=1}^{n}\left[-p b_{i i}(t) x_{i}^{p+1}+\left(1+p a_{i}(t)+\frac{p(p-1) \sigma_{i}^{2}(t)}{2}\right) x_{i}^{p}\right] \\
& +\sum_{i=1}^{n} \int_{\mathbb{Y}}\left[\left(1+\gamma_{i}(t, u)\right)^{p}-1-p \gamma_{i}(t, u)\right] \lambda(d u) x_{i}^{p} \\
\leq & K .
\end{aligned}
$$

Hence

$$
\mathbb{E}\left(e^{t} V(X(t))\right) \leq V\left(x_{0}\right)+\int_{0}^{t} K e^{s} d s=V\left(x_{0}\right)+K\left(e^{t}-1\right),
$$

which yields the desired assertion (3.3) by the inequality (2.2).

For any $p \in[0,1]$, according to the inequality $(2.1)$,

$$
\int_{\mathbb{Y}}\left[\left(1+\gamma_{i}(t, u)\right)^{p}-1-p \gamma_{i}(t, u)\right] \lambda(d u) \leq 0 .
$$


Consequently

$$
V(x)+\mathscr{L} V(x, t) \leq \sum_{i=1}^{n}\left[-p b_{i i}(t) x_{i}^{p+1}+\left(1+p a_{i}(t)\right) x_{i}^{p}\right],
$$

which has an upper bound by (A). Then (3.1) holds with $p \in[0,1]$ under (A).

Corollary 3.1. Under assumption (A), there exists an invariant probability measure for the solution $X(t)$ of Eq. (1.5).

Proof. Let $\mathbb{P}(t, x, A)$ be the transition probability measure of $X(t, x)$, starting from $x$ at time 0 . Define

$$
\mu_{T}(A):=\frac{1}{T} \int_{0}^{T} \mathbb{P}(t, x, A) d t
$$

and $B_{r}:=\left\{x \in \mathbb{R}_{+}^{n}:|x| \leq r\right\}$ for $r \geq 0$. In the light of Chebyshev's inequality and Theorem 3.1 with $p \in(0,1)$,

$$
\mu_{T}\left(B_{r}^{c}\right)=\frac{1}{T} \int_{0}^{T} \mathbb{P}\left(t, x, B_{r}^{c}\right) d t \leq \frac{1}{r^{p} T} \int_{0}^{T} \mathbb{E}|X(t, x)|^{p} d t \leq \frac{K}{r^{p}},
$$

and we have, for any $\epsilon>0, \mu_{T}\left(B_{r}\right)>1-\epsilon$ whenever $r$ is large enough. Hence $\left\{\mu_{T}, T>0\right\}$ is tight. By the Krylov-Bogoliubov theorem (see, e.g., [20, Corollary 3.1.2, p. 22]), the conclusion follows immediately.

Definition 3.1. The solution $X(t)$ of Eq. (1.5) is called stochastically bounded if for any $\epsilon \in(0,1)$, there is a constant $H:=H(\epsilon)$ such that for any $x_{0} \in \mathbb{R}_{+}^{n}$,

$$
\limsup _{t \rightarrow \infty} \mathbb{P}\{|X(t)| \leq H\} \geq 1-\epsilon .
$$

As an application of Theorem 3.1, together with the Chebyshev inequality, we can also establish the following corollary.

Corollary 3.2. Under assumption (A), the solution $X(t)$ of Eq. (1.5) is stochastically bounded.

For later applications, let us cite a strong law of large numbers for local martingales (see, e.g., Lipster [21]) as the following lemma.

Lemma 3.1. Let $M(t), t \geq 0$, be a local martingale vanishing at time 0 and define

$$
\rho_{M}(t):=\int_{0}^{t} \frac{d\langle M\rangle(s)}{(1+s)^{2}}, \quad t \geq 0,
$$

where $\langle M\rangle(t):=\langle M, M\rangle(t)$ is Meyer's angle bracket process. Then

$$
\lim _{t \rightarrow \infty} \frac{M(t)}{t}=0 \quad \text { a.s. provided that } \lim _{t \rightarrow \infty} \rho_{M}(t)<\infty \text { a.s. }
$$

Remark 3.1. Suppose that

$$
\Psi_{\mathrm{loc}}^{2}:=\left\{\Psi(t, z) \text { predictable }\left.\left|\int_{0}^{t} \int_{\mathbb{Y}}\right| \Psi(s, z)\right|^{2} \lambda(d u) d s<\infty\right\}
$$

and for $\Psi \in \Psi_{\mathrm{loc}}^{2}$,

$$
M(t):=\int_{0}^{t} \int_{\mathbb{Y}} \Psi(s, z) \tilde{N}(d s, d u) .
$$

Then, by, e.g., Kunita [22, Proposition 2.4],

$$
\langle M\rangle(t)=\int_{0}^{t} \int_{\mathbb{Y}}|\Psi(s, z)|^{2} \lambda(d u) d s \text { and }[M](t)=\int_{0}^{t} \int_{\mathbb{Y}}|\Psi(s, z)|^{2} N(d s, d u),
$$

where $[M](t):=[M, M](t)$, the square bracket process (or quadratic variation process) of $M(t)$.

Theorem 3.2. Let assumption (A) hold. Assume further that for some constant $\delta>-1$ and any $t \geq 0$,

$$
\gamma_{i}(t, u) \geq \delta, \quad u \in \mathbb{Y}, i=1, \ldots, n,
$$

and there exists a constant $K>0$ such that

$$
\int_{0}^{t} \int_{\mathbb{Y}}|\gamma(s, u)|^{2} \lambda(d u) d s \leq K t .
$$


Then the solution $X(t), t \geq 0$, of Eq. (1.5) has the property

$$
\limsup _{t \rightarrow \infty} \frac{1}{t}\left[\ln (|X(t)|)+\frac{\min _{1 \leq i \leq n} \hat{b}_{i i}}{\sqrt{n}} \int_{0}^{t}|X(s)| d s\right] \leq \max _{1 \leq i \leq n} \check{a}_{i}, \quad \text { a.s. }
$$

Proof. For any $x \in \mathbb{R}_{+}^{n}$, suppose that $V(x)=\sum_{i=1}^{n} x_{i}$; by Itô's formula,

$$
\begin{aligned}
\ln (V(X(t))) \leq & \ln \left(V\left(x_{0}\right)\right)+\int_{0}^{t}\left(X^{T}(s)(a(s)-B(s) X(s)) / V(X(s))-\left(X^{T}(s) \sigma(s)\right)^{2} /\left(2 V^{2}(X(s))\right)\right) d s \\
& +\int_{0}^{t} X^{T}(s) \sigma(s) / V(X(s)) d W(s)+\int_{0}^{t} \int_{\mathbb{Y}} \ln \left(1+H\left(X\left(s^{-}\right), s, u\right)\right) \tilde{N}(d s, d u),
\end{aligned}
$$

where

$$
H(x, t, u)=\left(\sum_{i=1}^{n} \gamma_{i}(t, u) x_{i}\right) / V(x) .
$$

Here we used the fact that $1+H>0$ and the inequality (2.3). Note from the inequality (2.2) and assumption (A) that

$$
\begin{aligned}
& X^{T}(s)(a(s)-B(s) X(s)) / V(X(s))-\left(X^{T}(s) \sigma(s)\right)^{2} /\left(2 V^{2}(X(s))\right) \leq \frac{\sum_{i=1}^{n} a_{i}(s) X_{i}(s)}{\sum_{i=1}^{n} X_{i}(s)}-\frac{\sum_{i=1}^{n} X_{i}(s) \sum_{j=1}^{n} b_{i j}(s) X_{j}(s)}{\sum_{i=1}^{n} X_{i}(s)} \\
& \quad \leq \max _{1 \leq i \leq n} \check{a}_{i}-\frac{\min _{1 \leq i \leq n} \hat{b}_{i i}}{\sqrt{n}}|X(s)| .
\end{aligned}
$$

Suppose that

$$
M(t):=\int_{0}^{t} X^{T}(s) \sigma(s) / V(X(s)) d W(s) \quad \text { and } \quad \tilde{M}(t):=\int_{0}^{t} \int_{\mathbb{Y}} \ln \left(1+H\left(X\left(s^{-}\right), s, u\right)\right) \tilde{N}(d s, d u) .
$$

Compute by the boundedness of $\sigma$ that

$$
\langle M\rangle(t)=\int_{0}^{t}\left(X^{T}(s) \sigma(s)\right)^{2} / V^{2}(X(s)) d s \leq \int_{0}^{t}|\sigma(s)|^{2} d s \leq K t .
$$

On the other hand, by assumption (3.6) and the definition of $H$, for $x \in \mathbb{R}_{+}^{n}$ we obtain

$$
H(x, t, u) \geq \delta
$$

and, in addition to (2.3), for $-1<\delta \leq 0$,

$$
\begin{aligned}
|\ln (1+H(x, t, u))| & \leq\left|\ln (1+H(x, y, u)) I_{\{\delta \leq H(x, t, u) \leq 0\}}\right|+\left|\ln (1+H(x, y, u)) I_{\{0 \leq H(x, t, u)\}}\right| \\
& \leq-\ln (1+\delta)+|H(x, t, u)| .
\end{aligned}
$$

This, together with (3.7), gives that

$$
\begin{aligned}
\langle\tilde{M}\rangle(t) & =\int_{0}^{t} \int_{\mathbb{Y}}(\ln (1+H(X(s), s, u)))^{2} \lambda(d u) d s \\
& \leq 2(-\ln (1+\delta))^{2} \lambda(\mathbb{Y}) t+2 \int_{0}^{t} \int_{\mathbb{Y}} H^{2}(X(s), s, u) \lambda(d u) d s \\
& \leq 2(-\ln (1+\delta))^{2} \lambda(\mathbb{Y}) t+2 \int_{0}^{t} \int_{\mathbb{Y}}|\gamma(t, u)|^{2} \lambda(d u) d s \\
& \leq\left(2(-\ln (1+\delta))^{2} \lambda(\mathbb{Y})+K\right) t .
\end{aligned}
$$

Then the strong law of large numbers, Lemma 3.1, yields

$$
\frac{1}{t} M(t) \rightarrow 0 \text { a.s. and } \frac{1}{t} \tilde{M}(t) \rightarrow 0 \text { as } t \rightarrow \infty,
$$

and the conclusion follows. 


\section{The variation-of-constants formula and the sample Lyapunov exponents}

In this part we further discuss the long-term behaviours of model (1.5). To begin, we obtain the following variation-ofconstants formula for one-dimensional diffusion with jumps, which is interesting in its own right.

\subsection{The variation-of-constants formula}

Lemma 4.1. Let $F, G, f, g: \mathbb{R}_{+} \rightarrow \mathbb{R}$ and $H, h: \mathbb{R}_{+} \times \mathbb{Y} \rightarrow \mathbb{R}$ be Borel measurable and bounded functions with property $H>-1$, and $Y(t)$ satisfy

$$
\begin{aligned}
& d Y(t)=[F(t) Y(t)+f(t)] d t+[G(t) Y(t)+g(t)] d W(t)+\int_{\mathbb{Y}}\left[Y\left(t^{-}\right) H(t, u)+h(t, u)\right] \tilde{N}(d t, d u), \\
& Y(0)=Y_{0} .
\end{aligned}
$$

Then the solution can be explicitly expressed as

$$
\begin{aligned}
Y(t)= & \Phi(t)\left(Y_{0}+\int_{0}^{t} \Phi^{-1}(s)\left[\left(f(s)-G(s) g(s)-\int_{\mathbb{Y}} \frac{H(s, u) h(s, u)}{1+H(s, u)} \lambda(d u)\right) d s\right.\right. \\
& \left.\left.+g(s) d W(s)+\int_{\mathbb{Y}} \frac{h(s, u)}{1+H(s, u)} \tilde{N}(d s, d u)\right]\right),
\end{aligned}
$$

where

$$
\begin{aligned}
\Phi(t):= & \exp \left[\int_{0}^{t}\left(F(s)-\frac{1}{2} G^{2}(s)+\int_{\mathbb{Y}}[\ln (1+H(s, u))-H(s, u)] \lambda(d u)\right) d s\right. \\
& \left.+\int_{0}^{t} G(s) d W(s)+\int_{0}^{t} \int_{\mathbb{Y}} \ln (1+H(s, u)) \tilde{N}(d s, d u)\right]
\end{aligned}
$$

is the fundamental solution of the corresponding homogeneous linear equation

$$
d Z(t)=F(t) Z(t) d t+G(t) Z(t) d W(t)+Z\left(t^{-}\right) \int_{\mathbb{Y}} H(t, u) \tilde{N}(d t, d u) .
$$

Proof. Noting that

$$
\begin{aligned}
\Phi(t)= & \exp \left[\int_{0}^{t}\left(F(s)-\frac{1}{2} G^{2}(s)+\int_{\mathbb{Y}}[\ln (1+H(s, u))-H(s, u)] \lambda(d u)\right) d s\right. \\
& \left.+\int_{0}^{t} G(s) d W(s)+\int_{0}^{t} \int_{\mathbb{Y}} \ln (1+H(s, u)) \tilde{N}(d s, d u)\right]
\end{aligned}
$$

is the fundamental solution to Eq. (4.2), we then have

$$
d \Phi(t)=F(t) \Phi(t) d t+G(t) \Phi(t) d W(t)+\Phi\left(t^{-}\right) \int_{\mathbb{Y}} H(t, u) \tilde{N}(d t, d u) .
$$

By [23, Theorem 1.19, p. 10], Eq. (4.1) has a unique solution $Y(t), t \geq 0$. We assume that

$$
Y(t)=\Phi(t)\left(Y(0)+\int_{0}^{t} \Phi^{-1}(s)\left[\bar{f}(s) d s+\bar{g}(s) d W(s)+\int_{\mathbb{Y}} \bar{h}(s, u) \tilde{N}(d s, d u)\right]\right),
$$

where $\bar{f}, \bar{g}$, and $\bar{h}$ are functions to be determined. Suppose that

$$
\bar{Y}(t)=Y(0)+\int_{0}^{t} \Phi^{-1}(s)\left[\bar{f}(s) d s+\bar{g}(s) d W(s)+\int_{\mathbb{Y}} \bar{h}(s, u) \tilde{N}(d s, d u)\right],
$$

which means that

$$
d \bar{Y}(t)=\Phi^{-1}(t)\left[\bar{f}(t) d t+\bar{g}(t) d W(t)+\int_{\mathbb{Y}} \bar{h}(t, u) \tilde{N}(d t, d u)\right] .
$$

Observing that $\Phi$ and $\bar{Y}$ are real-valued Lévy-type stochastic integrals, by Itô's product formula (see, e.g., [24, Theorem 4.4.13, p. 231]), we can deduce that

$$
d Y(t)=\Phi\left(t^{-}\right) d \bar{Y}(t)+\bar{Y}\left(t^{-}\right) d \Phi(t)+d[\Phi, \bar{Y}](t)
$$


where $[\Phi, \bar{Y}]$ is the cross-quadratic variation of processes $\Phi$ and $\bar{Y}$, and by (4.14) in [24, p. 230],

$$
d[\Phi, \bar{Y}](t)=G(t) \bar{g}(t) d t+\int_{\mathbb{Y}} H(t, u) \bar{h}(t, u) N(d t, d u) .
$$

Putting (4.3), (4.4) and (4.6) into (4.5), we deduce that

$$
\begin{aligned}
d Y(t)= & {\left[\bar{f}(t) d t+\bar{g}(t) d W(t)+\int_{\mathbb{Y}} \bar{h}(t, u) \tilde{N}(d t, d u)\right] } \\
& +F(t) Y(t) d t+G(t) Y(t) d W(t)+Y\left(t^{-}\right) \int_{\mathbb{Y}} H(t, u) \tilde{N}(d t, d u) \\
& +G(t) \bar{g}(t) d t+\int_{\mathbb{Y}} H(t, u) \bar{h}(t, u) N(d t, d u) \\
= & {\left[\bar{f}(t)+F(t) Y(t)+G(t) \bar{g}(t)+\int_{\mathbb{Y}} H(t, u) \bar{h}(t, u) \lambda(d u)\right] d t+[\bar{g}(t)+G(t) Y(t)] d W(t) } \\
& +\int_{\mathbb{Y}}\left[\bar{h}(t, u)+Y\left(t^{-}\right) H(t, u)+H(t, u) \bar{h}(t, u)\right] \tilde{N}(d t, d u) .
\end{aligned}
$$

Setting

$$
\bar{f}(t)+G(t) \bar{g}(t)+\int_{\mathbb{Y}} H(t, u) \bar{h}(t, u) \lambda(d u)=f(t)
$$

and

$$
\bar{g}(t)=g(t) \text { and } \bar{h}(t, u)+H(t, u) \bar{h}(t, u)=h(t, u),
$$

we hence derive that

$$
\bar{f}(t)=f(t)-G(t) g(t)-\int_{\mathbb{Y}} \frac{H(t, u) h(t, u)}{1+H(t, u)} \lambda(d u), \quad \bar{g}(t)=g(t) \text { and } \bar{h}(t, u)=\frac{h(t, u)}{1+H(t, u)}
$$

and the required expression follows.

\subsection{The one-dimensional competitive model}

In what follows, we shall study some properties of the processes $Y_{i}(t)$ defined by (2.4), which is actually a onedimensional competitive model.

Lemma 4.2. Under assumption (A), Eq. (2.4) admits a unique positive solution $Y_{i}(t), t \geq 0$, which admits the explicit formula

$$
Y_{i}(t)=\frac{\Phi_{i}(t)}{\frac{1}{X_{i}(0)}+\int_{0}^{t} \Phi_{i}(s) b_{i i}(s) d s},
$$

where

$$
\begin{aligned}
\Phi_{i}(t):= & \exp \left(\int_{0}^{t}\left[a_{i}(s)-\frac{1}{2} \sigma_{i}^{2}(s)+\int_{\mathbb{Y}}\left(\ln \left(1+\gamma_{i}(s, u)\right)-\gamma_{i}(s, u)\right) \lambda(d u)\right] d s\right. \\
& \left.+\int_{0}^{t} \sigma_{i}(s) d W(s)+\int_{0}^{t} \int_{\mathbb{Y}} \ln \left(1+\gamma_{i}(s, u)\right) \tilde{N}(d s, d u)\right) .
\end{aligned}
$$

Proof. It is easy to see that $\Phi_{i}(t)$ is integrable in any finite interval; hence $Y_{i}(t)$ will never reach 0 . Supposing that $\bar{Y}_{i}(t):=\frac{1}{Y_{i}(t)}$ and applying the Itô formula, we have

$$
\begin{aligned}
d \bar{Y}_{i}(t)= & -\frac{1}{Y_{i}^{2}(t)} Y_{i}(t)\left[\left(a_{i}(t)-b_{i i}(t) Y_{i}(t)\right) d t+\sigma_{i}(t) d W(t)\right]+\frac{1}{2} \frac{2}{Y_{i}^{3}(t)} \sigma_{i}^{2}(t) Y_{i}^{2}(t) d t \\
& +\int_{\mathbb{Y}}\left[\frac{1}{\left(1+\gamma_{i}(t, u)\right) Y_{i}(t)}-\frac{1}{Y_{i}(t)}+\frac{1}{Y_{i}^{2}(t)} Y_{i}(t) \gamma_{i}(t, u)\right] \lambda(d u) d t \\
& +\int_{\mathbb{Y}}\left[\frac{1}{\left(1+\gamma_{i}(t, u)\right) Y_{i}\left(t^{-}\right)}-\frac{1}{Y_{i}\left(t^{-}\right)}\right] \tilde{N}(d t, d u),
\end{aligned}
$$


that is,

$$
\begin{aligned}
d \bar{Y}(t)= & \bar{Y}\left(t^{-}\right)\left[\left(\sigma_{i}^{2}(t)-a_{i}(t)+\int_{\mathbb{Y}}\left(\frac{1}{1+\gamma_{i}(t, u)}-1+\gamma_{i}(t, u)\right) \lambda(d u)\right) d t-\sigma_{i}(t) d W(t)\right. \\
& \left.+\int_{\mathbb{Y}}\left(\frac{1}{1+\gamma_{i}(t, u)}-1\right) \tilde{N}(d t, d u)\right]+b_{i i}(t) d t .
\end{aligned}
$$

By Lemma 4.1, Eq. (4.8) has an explicit solution and the conclusion Eq. (4.7) follows.

Definition 4.1. The solution of Eq. (2.4) is said to be stochastically permanent if for any $\epsilon \in(0,1)$ there exist positive constants $H_{1}:=H_{1}(\epsilon)$ and $H_{2}:=H_{2}(\epsilon)$ such that

$$
\liminf _{t \rightarrow \infty} \mathbb{P}\left\{Y_{i}(t) \leq H_{1}\right\} \geq 1-\epsilon \text { and } \liminf _{t \rightarrow \infty} \mathbb{P}\left\{Y_{i}(t) \geq H_{2}\right\} \geq 1-\epsilon .
$$

Theorem 4.1. Let assumption (A) hold. Assume further that there exists a constant $c_{1}>0$ such that, for any $t \geq 0$ and $i=$ $1, \ldots, n$,

$$
a_{i}(t)-\sigma_{i}^{2}(t)-\int_{\mathbb{Y}} \frac{\gamma_{i}^{2}(t, u)}{1+\gamma_{i}(t, u)} \lambda(d u) \geq c_{1} .
$$

Then the solution $Y_{i}(t), t \geq 0$, of Eq. (2.4) is stochastically permanent.

Proof. The first part of the proof follows by the Chebyshev inequality and Corollary 3.2. Observe that (4.7) can be rewritten in the form

$$
\begin{aligned}
\frac{1}{Y_{i}(t)}= & \frac{1}{X_{i}(0)} \exp \left(\int_{0}^{t}-\left[a_{i}(s)-\frac{1}{2} \sigma_{i}^{2}(s)+\int_{\mathbb{Y}}\left(\ln \left(1+\gamma_{i}(s, u)\right)-\gamma_{i}(s, u)\right) \lambda(d u)\right] d s\right. \\
& \left.-\int_{0}^{t} \sigma_{i}(s) d W(s)-\int_{0}^{t} \int_{\mathbb{Y}} \ln \left(1+\gamma_{i}(s, u)\right) \tilde{N}(d s, d u)\right) \\
& +\int_{0}^{t} b_{i i}(s) \exp \left(\int_{s}^{t}-\left[a(r)-\frac{1}{2} \sigma_{i}^{2}(r)+\int_{\mathbb{Y}}\left(\ln \left(1+\gamma_{i}(r, u)\right)-\gamma_{i}(r, u)\right) \lambda(d u)\right] d r\right. \\
& \left.-\int_{s}^{t} \sigma_{i}(r) d W(r)-\int_{s}^{t} \int_{\mathbb{Y}} \ln \left(1+\gamma_{i}(r, u)\right) \tilde{N}(d r, d u)\right) d s .
\end{aligned}
$$

By, e.g., [24, Corollary 5.2.2, p. 253], we note that

$$
\begin{aligned}
\exp & \left(-\frac{1}{2} \int_{0}^{t} \sigma_{i}^{2}(s) d s-\int_{0}^{t} \int_{\mathbb{Y}}\left(\frac{1}{1+\gamma_{i}(s, u)}-1+\ln \left(1+\gamma_{i}(s, u)\right)\right) \lambda(d u) d s\right. \\
& \left.-\int_{0}^{t} \sigma_{i}(s) d W(s)-\int_{0}^{t} \int_{\mathbb{Y}} \ln \left(1+\gamma_{i}(s, u)\right) \tilde{N}(d s, d u)\right)
\end{aligned}
$$

is a local martingale. Hence supposing that $\bar{M}_{i}(t):=\frac{1}{Y_{i}(t)}$ and taking expectations on both sides of (4.10) leads to

$$
\begin{aligned}
\mathbb{E} \bar{M}_{i}(t)= & \frac{1}{X_{i}(0)} \exp \left(-\int_{0}^{t}\left[a_{i}(s)-\sigma_{i}^{2}(s)-\int_{\mathbb{Y}} \frac{\gamma_{i}^{2}(s, u)}{1+\gamma_{i}(s, u)} \lambda(d u)\right]\right) d s \\
& +\int_{0}^{t} b_{i i}(s) \exp \left(-\int_{s}^{t}\left[a_{i}(r)-\sigma_{i}^{2}(r)-\int_{\mathbb{Y}} \frac{\gamma_{i}^{2}(r, u)}{1+\gamma_{i}(r, u)} \lambda(d u)\right]\right) d r d s,
\end{aligned}
$$

which, combining with (4.9), yields

$$
\mathbb{E} \bar{M}_{i}(t) \leq \frac{1}{X_{i}(0)} e^{-c_{1} t}+\int_{0}^{t} b_{i i}(s) e^{-c_{2}(t-s)} d s \leq \frac{\check{b}}{c_{1}}+\left(\frac{1}{X_{i}(0)}-\frac{\check{b}}{c_{1}}\right) e^{-c_{1} t} .
$$

Hence there exists a constant $K>0$ such that

$$
\mathbb{E} \bar{M}_{i}(t) \leq K \text {. }
$$

Furthermore, for any $\epsilon>0$ and constant $H_{2}(\epsilon)>0$, thanks to the Chebyshev inequality and (4.12),

$$
\mathbb{P}\left\{Y_{i}(t) \geq H_{2}\right\}=\mathbb{P}\left\{\bar{M}_{i}(t) \leq 1 / H_{2}\right\}=1-\mathbb{P}\left\{\bar{M}_{i}(t)>1 / H_{2}\right\} \geq 1-H_{2} \mathbb{E} \bar{M}_{i}(t) \geq 1-\epsilon
$$

whenever $H_{2}=\epsilon / K$, as required. 
Theorem 4.2. Let the conditions of Theorem 4.1 hold. Then Eq. (2.4) has the property

$$
\lim _{t \rightarrow \infty} \mathbb{E}\left|Y_{i}(t, x)-Y_{i}(t, y)\right|^{\frac{1}{2}}=0 \quad \text { uniformly in }(x, y) \in \mathbb{K} \times \mathbb{K},
$$

where $\mathbb{K}$ is any compact subset of $(0, \infty)$.

Proof. By the Hölder inequality,

$$
\begin{aligned}
\mathbb{E}\left|Y_{i}(t, x)-Y_{i}(t, y)\right|^{\frac{1}{2}} & =\mathbb{E}\left(Y_{i}(t, x) Y_{i}(t, y)\left|\frac{1}{Y_{i}(t, y)}-\frac{1}{Y_{i}(t, x)}\right|\right)^{\frac{1}{2}} \\
& \leq\left(\mathbb{E}\left(Y_{i}(t, x) Y_{i}(t, y)\right)\right)^{\frac{1}{2}}\left(\mathbb{E}\left|\frac{1}{Y_{i}(t, y)}-\frac{1}{Y_{i}(t, x)}\right|\right)^{\frac{1}{2}} .
\end{aligned}
$$

To show the desired assertion it is sufficient to estimate the two terms on the right-hand side of the last step. By virtue of the Itô formula,

$$
\begin{aligned}
d\left(Y_{i}(t, x) Y_{i}(t, y)\right)= & Y_{i}\left(t^{-}, x\right) d Y_{i}(t, y)+Y_{i}\left(t^{-}, y\right) d Y_{i}(t, x)+d\left[Y_{i}(t, x), Y_{i}(t, y)\right] \\
= & Y_{i}\left(t^{-}, x\right) Y_{i}\left(t^{-}, y\right)\left[\left(a_{i}(t)-b_{i i}(t) Y_{i}\left(t^{-}, y\right)\right) d t+\sigma_{i}(t) d W(t)+\int_{\mathbb{Y}} \gamma_{i}(t, u) \tilde{N}(d t, d u)\right] \\
& +Y_{i}\left(t^{-}, x\right) Y_{i}\left(t^{-}, y\right)\left[\left(a_{i}(t)-b_{i i}(t) Y_{i}\left(t^{-}, x\right)\right) d t+\sigma_{i}(t) d W(t)+\int_{\mathbb{Y}} \gamma_{i}(t, u) \tilde{N}(d t, d u)\right] \\
& +\sigma_{i}^{2}(t) Y_{i}(t, x) Y_{i}(t, y) d t+\int_{\mathbb{Y}} \gamma_{i}^{2}(t, u) Y_{i}\left(t^{-}, x\right) Y_{i}\left(t^{-}, y\right) N(d t, d u) \\
= & \left(2 a_{i}(t)+\sigma_{i}^{2}(t)\right) Y_{i}(t, x) Y_{i}(t, y) d t-b_{i i}(t) Y_{i}(t, x) Y_{i}(t, y)\left(Y_{i}(t, x)+Y_{i}(t, y)\right) d t \\
& +2 \sigma_{i}(t) Y_{i}(t, x) Y_{i}(t, y) d W(t)+2 \int_{\mathbb{Y}} \gamma_{i}(t, u) Y_{i}\left(t^{-}, x\right) Y_{i}\left(t^{-}, y\right) \tilde{N}(d t, d u) \\
& +\int_{\mathbb{Y}} \gamma_{i}^{2}(t, u) Y_{i}\left(t^{-}, x\right) Y_{i}\left(t^{-}, y\right) N(d t, d u) .
\end{aligned}
$$

Thus, in view of Jensen's inequality and the familiar inequality $a+b \geq 2 \sqrt{a b}$ for any $a, b \geq 0$, we deduce that

$$
\begin{aligned}
\mathbb{E}\left(Y_{i}(t, x) Y_{i}(t, y)\right) \leq & x y+\int_{0}^{t} \delta_{i}(s) \mathbb{E}\left(Y_{i}(s, x) Y_{i}(s, y)\right) d s \\
& -\mathbb{E} \int_{0}^{t} b_{i i}(s)\left(Y_{i}(s, x) Y_{i}(s, y)\left(Y_{i}(s, x)+Y_{i}(s, y)\right)\right) d s \\
\leq & x y+\int_{0}^{t} \delta_{i}(s) \mathbb{E}\left(Y_{i}(s, x) Y_{i}(s, y)\right) d s-\int_{0}^{t} b_{i i}(s)\left(\mathbb{E}\left(Y_{i}(s, x) Y_{i}(s, y)\right)\right)^{\frac{3}{2}} d s,
\end{aligned}
$$

where $\delta_{i}(t):=2 a_{i}(t)+\sigma_{i}^{2}(t)+\int_{\mathbb{Y}} \gamma_{i}^{2}(t, u) \lambda(d u)$. By the comparison theorem,

$$
\begin{aligned}
\mathbb{E}\left(Y_{i}(t, x) Y_{i}(t, y)\right) & \leq\left(1 / \sqrt{x y} e^{-\frac{1}{2} \int_{0}^{t} \delta_{i}(s) d s}+\frac{1}{2} \int_{0}^{t} b_{i i}(s) e^{-\frac{1}{2} \int_{s}^{t} \delta_{i}(\tau) d \tau} d s\right)^{-2} \\
& \leq\left(\hat{b} / \check{\delta}_{i}+\left(1 / \sqrt{x y}-\hat{b}_{i i} / \check{\delta}_{i}\right) e^{-\frac{\check{\delta}_{i} t}{2}}\right)^{-2} .
\end{aligned}
$$

On the other hand, thanks to (4.7) we have

$$
\begin{aligned}
\frac{1}{Y_{i}(t, x)}-\frac{1}{Y_{i}(t, y)}= & \left(\frac{1}{x}-\frac{1}{y}\right) \exp \left(-\int_{0}^{t}\left[a_{i}(s)-\frac{1}{2} \sigma_{i}^{2}(s)+\int_{\mathbb{Y}}\left(\ln \left(1+\gamma_{i}(s, u)\right)-\gamma_{i}(s, u)\right) \lambda(d u)\right] d s\right. \\
& \left.-\int_{0}^{t} \sigma_{i}(s) d W(s)-\int_{0}^{t} \int_{\mathbb{Y}} \ln \left(1+\gamma_{i}(s, u)\right) \tilde{N}(d s, d u)\right)
\end{aligned}
$$

In the same way as (4.11) was treated, it follows from (4.9) that

$$
\mathbb{E}\left|\frac{1}{Y_{i}(t, x)}-\frac{1}{Y_{i}(t, y)}\right| \leq\left|\frac{1}{x}-\frac{1}{y}\right| e^{-c_{2} t} .
$$

Thus (4.13) follows on combining (4.14) and (4.15). 
If $a_{i}, b_{i i}, \sigma_{i}, \gamma_{i}$ are time independent, Eq. (2.4) reduces to

$$
d Y_{i}(t)=Y_{i}\left(t^{-}\right)\left[\left(a_{i}-b_{i i} Y_{i}(t)\right) d t+\sigma_{i} d W(t)+\int_{\mathbb{Y}} \gamma_{i}(u) \tilde{N}(d t, d u)\right],
$$

with original value $x>0$. Let $p(t, x, d y)$ denote the transition probability of solution process $Y_{i}(t, x)$ and $\mathbb{P}(t, x, A)$ denote the probability of event $\left\{Y_{i}(t, x) \in A\right\}$, where $A$ is a Borel measurable subset of $(0, \infty)$. This is similar to the case of Corollary 3.1; under the conditions of Theorem 4.1 there exists an invariant measure for $Y_{i}(t, x)$. Moreover by the standard procedure [11, pp. 213-216], we know that Theorem 4.2 implies the uniqueness of the invariant measure. That is:

Theorem 4.3. Under the conditions of Theorems 4.1 and 4.2, the solution $Y_{i}(t, x)$ of Eq. (4.16) has a unique invariant measure.

We further need the following exponential martingale inequality with jumps; see, e.g., [24, Theorem 5.2.9, p. 291].

Lemma 4.3. Assume that $g:[0, \infty) \rightarrow \mathbb{R}$ and $h:[0, \infty) \times \mathbb{Y} \rightarrow \mathbb{R}$ are both predictable $\mathcal{F}_{t}$-adapted processes such that for any $T>0$,

$$
\int_{0}^{T}|g(t)|^{2} d t<\infty \quad \text { a.s. and } \int_{0}^{T} \int_{\mathbb{Y}}|h(t, u)|^{2} \lambda(d u) d t<\infty \quad \text { a.s. }
$$

Then for any constants $\alpha, \beta>0$,

$$
\begin{gathered}
\mathbb{P}\left\{\operatorname { s u p } _ { 0 \leq t \leq T } \left[\int_{0}^{t} g(s) d W(s)-\frac{\alpha}{2} \int_{0}^{t}|g(s)|^{2} d s+\int_{0}^{t} \int_{\mathbb{Y}} h(s, u) \tilde{N}(d s, d u)\right.\right. \\
\left.\left.-\frac{1}{\alpha} \int_{0}^{t} \int_{\mathbb{Y}}\left[e^{\alpha h(s, u)}-1-\alpha h(s, u)\right] \lambda(d u) d s\right]>\beta\right\} \leq e^{-\alpha \beta} .
\end{gathered}
$$

Lemma 4.4. Let assumption (A) hold. Assume further that for any $t \geq 0$ and $i=1, \ldots, n$,

$$
\sup _{t \geq 0} \int_{0}^{t} \int_{\mathbb{Y}} e^{s-t}\left[\gamma_{i}(s, u)-\ln \left(1+\gamma_{i}(s, u)\right)\right] \lambda(d u) d s<\infty .
$$

Then

$$
\limsup _{t \rightarrow \infty} \frac{\ln Y_{i}(t)}{\ln t} \leq 1, \quad \text { a.s. for each } i=1, \ldots, n .
$$

Proof. For any $t \geq 0$ and $i=1, \ldots, n$, applying the Itô formula we have

$$
\begin{aligned}
e^{t} \ln Y_{i}(t)= & \ln X_{i}(0)+\int_{0}^{t} e^{s}\left[\ln Y_{i}(s)+a_{i}(s)-b_{i i}(s) Y_{i}(s)-\frac{1}{2} \sigma_{i}^{2}(s)+\int_{\mathbb{Y}}\left[\ln \left(1+\gamma_{i}(s, u)\right)-\gamma_{i}(s, u)\right] \lambda(d u)\right] d s \\
& +\int_{0}^{t} e^{s} \sigma_{i}(s) d W(s)+\int_{0}^{t} \int_{\mathbb{Y}} e^{s} \ln \left(1+\gamma_{i}(s, u)\right) \tilde{N}(d s, d u)
\end{aligned}
$$

Note that, for $c, x>0, \ln x-c x$ attains its maximum value $-1-\ln c$ at $x=\frac{1}{c}$. Thus it follows from the inequality (2.3) that

$$
\begin{aligned}
e^{t} \ln Y_{i}(t) \leq & \ln X_{i}(0)+\int_{0}^{t} e^{s}\left[-1-\ln b_{i i}(s)+a_{i}(s)-\frac{1}{2} \sigma_{i}^{2}(s)\right] d s \\
& +\int_{0}^{t} e^{s} \sigma_{i}(s) d W(s)+\int_{0}^{t} \int_{\mathbb{Y}} e^{s} \ln \left(1+\gamma_{i}(s, u)\right) \tilde{N}(d s, d u) .
\end{aligned}
$$

In the light of Lemma 4.3, for any $\alpha, \beta, T>0$,

$$
\begin{array}{r}
\mathbb{P}\left\{\operatorname { s u p } _ { 0 \leq t \leq T } \left[\int_{0}^{t} e^{s} \sigma_{i}(s) d W(s)-\frac{\alpha}{2} \int_{0}^{t} e^{2 s} \sigma_{i}^{2}(s) d s+\int_{0}^{t} \int_{\mathbb{Y}} e^{s} \ln \left(1+\gamma_{i}(s, u)\right) \tilde{N}(d s, d u)\right.\right. \\
\left.\left.-\frac{1}{\alpha} \int_{0}^{t} \int_{\mathbb{Y}}\left[e^{\alpha e^{s} \ln \left(1+\gamma_{i}(s, u)\right)}-1-\alpha e^{s} \ln \left(1+\gamma_{i}(s, u)\right)\right] \lambda(d u) d s\right] \geq \beta\right\} \leq e^{-\alpha \beta} .
\end{array}
$$

Choose $T=k \gamma, \alpha=\epsilon e^{-k \gamma}$, and $\beta=\frac{\theta e^{k \gamma} \ln k}{\epsilon}$, where $k \in \mathbb{N}, 0<\epsilon<1, \gamma>0$, and $\theta>1$ in the above equation. Since $\sum_{k=1}^{\infty} k^{-\theta}<\infty$, we can deduce from the Borel-Cantelli lemma that there exists an $\Omega_{i} \subseteq \Omega$ with $\mathbb{P}\left(\Omega_{i}\right)=1$ such that for 
any $\epsilon \in \Omega_{i}$, an integer $k_{i}=k_{i}(\omega, \epsilon)$ can be found such that

$$
\begin{gathered}
\int_{0}^{t} e^{s} \sigma_{i}(s) d W(s)+\int_{0}^{t} \int_{\mathbb{Y}} e^{s} \ln \left(1+\gamma_{i}(s, u)\right) \tilde{N}(d s, d u) \leq \frac{\theta e^{k \gamma} \ln k}{\epsilon}+\frac{\epsilon e^{-k \gamma}}{2} \int_{0}^{t} e^{2 s} \sigma_{i}^{2}(s) d s \\
+\frac{1}{\epsilon e^{-k \gamma}} \int_{0}^{t} \int_{\mathbb{Y}}\left[\left(1+\gamma_{i}(s, u)\right)^{\epsilon e^{s-k \gamma}}-1-\epsilon e^{s-k \gamma} \ln \left(1+\gamma_{i}(s, u)\right)\right] \lambda(d u) d s
\end{gathered}
$$

whenever $k \geq k_{i}, 0 \leq t \leq k \gamma$. Next, note from the inequality (2.1) that, for any $\omega \in \Omega_{i}$ and $0<\epsilon<1,0 \leq t \leq k \gamma$ with $k \geq k_{i}$,

$$
\begin{aligned}
& \frac{1}{\epsilon e^{t-k \gamma}} \int_{0}^{t} \int_{\mathbb{Y}}^{t}\left[\left(1+\gamma_{i}(s, u)\right)^{\epsilon e^{s-k \gamma}}-1-\epsilon e^{s-k \gamma} \ln \left(1+\gamma_{i}(s, u)\right)\right] \lambda(d u) d s \\
& \quad \leq \int_{0}^{t} \int_{\mathbb{Y}} e^{s-t}\left(\gamma_{i}(s, u)-\ln \left(1+\gamma_{i}(s, u)\right)\right) \lambda(d u) d s .
\end{aligned}
$$

Thus, for $\omega \in \Omega_{i}$ and $(k-1) \gamma \leq t \leq k \gamma$ with $k \geq k_{i}+1$, we have

$$
\begin{aligned}
\frac{\ln Y_{i}(t)}{\ln t} \leq & \frac{\ln X_{i}(0)}{e^{t} \ln t}+\frac{\theta e^{k \gamma} \ln k}{\epsilon e^{(k-1) \gamma} \ln ((k-1) \gamma)}+\frac{1}{\ln t} \int_{0}^{t} e^{s-t}\left[-1-\ln b_{i i}(s)+a_{i}(s)-\frac{1}{2}\left(1-\epsilon e^{s-k \gamma}\right) \sigma_{i}^{2}(s)\right] d s \\
& +\frac{1}{\ln t} \int_{0}^{t} \int_{\mathbb{Y}} e^{s-t}\left[\gamma_{i}(s, u)-\ln \left(1+\gamma_{i}(s, u)\right)\right] \lambda(d u) d s .
\end{aligned}
$$

Supposing that $k \uparrow \infty$, combining assumption (A) and (4.17) leads to

$$
\limsup _{t \rightarrow \infty} \frac{\ln Y_{i}(t)}{\ln t} \leq \frac{\theta e^{\gamma}}{\epsilon},
$$

and the conclusion follows on setting $\gamma \downarrow 0, \epsilon \uparrow 1$, and $\theta \downarrow 1$.

Noting the $\operatorname{limit}_{\lim _{t \rightarrow \infty}} \frac{\ln t}{t}=0$, we have the following corollary.

Corollary 4.1. Under the conditions of Lemma 4.4,

$$
\limsup _{t \rightarrow \infty} \frac{\ln Y_{i}(t)}{t} \leq 0, \quad \text { a.s. for each } i=1, \ldots, n,
$$

and therefore

$$
\limsup _{t \rightarrow \infty} \frac{\ln \left(\prod_{i=1}^{n} Y_{i}(t)\right)}{t} \leq 0, \quad \text { a.s. }
$$

Corollary 4.2. Under the conditions of Lemma 4.4,

$$
\limsup _{t \rightarrow \infty} \frac{\ln \left(X_{i}(t)\right)}{t} \leq 0, \quad \text { a.s. for each } i=1, \ldots, n,
$$

and therefore

$$
\limsup _{t \rightarrow \infty} \frac{\ln \left(\prod_{i=1}^{n} X_{i}(t)\right)}{t} \leq 0, \quad \text { a.s. }
$$

Proof. Recalling

$$
Z_{i}(t) \leq X_{i}(t) \leq Y_{i}(t), \quad t \geq 0, i=1, \ldots, n
$$

and combining Corollary 4.1, we complete the proof.

Theorem 4.4. Let the conditions of Lemma 4.4 hold. Assume further that for any $t \geq 0$ and $i=1, \ldots, n$,

$$
R_{i}(t):=a_{i}(t)-\frac{1}{2} \sigma^{2}(t)+\int_{\mathbb{Y}}\left(\ln \left(1+\gamma_{i}(t, u)\right)-\gamma_{i}(t, u)\right) \lambda(d u) \geq 0,
$$

and there exists a constant $c_{2}>0$ such that

$$
\int_{\mathbb{Y}}\left(\ln \left(1+\gamma_{i}(t, u)\right)\right)^{2} \lambda(d u) \leq c_{2} .
$$


Then for each $i=1, \ldots, n$,

$$
\lim _{t \rightarrow \infty} \frac{\ln Y_{i}(t)}{t}=0 \quad \text { a.s. }
$$

Proof. According to Corollary 4.1, it suffices to show that $\lim _{\inf } \rightarrow \infty \frac{\ln Y_{i}(t)}{t} \geq 0$. Define for $t \geq 0$

$$
M_{i}(t):=\int_{0}^{t} \sigma_{i}(s) d W(s) \quad \text { and } \quad \bar{M}_{i}(t):=\int_{0}^{t} \int_{\mathbb{Y}} \ln \left(1+\gamma_{i}(s, u)\right) \tilde{N}(d s, d u) .
$$

Note that

$$
\left[M_{i}\right](t)=\left\langle M_{i}\right\rangle(t)=\int_{0}^{t} \sigma_{i}^{2}(s) d s \leq \check{\sigma}_{i}^{2} t
$$

and by (4.20),

$$
\left\langle\bar{M}_{i}\right\rangle(t)=\int_{0}^{t} \int_{\mathbb{Y}}\left(\ln \left(1+\gamma_{i}(s, u)\right)\right)^{2} \lambda(d u) d s \leq c_{2} t .
$$

We have

$$
\int_{0}^{t} \frac{1}{(1+s)^{2}} d s=-\left.\frac{1}{1+s}\right|_{0} ^{t}=\frac{t}{1+t}<\infty
$$

and combining this with Lemma 3.1, we then obtain

$$
\lim _{t \rightarrow \infty} \frac{1}{t} \int_{0}^{t} \sigma_{i}(s) d W(s)=0 \quad \text { a.s. and } \lim _{t \rightarrow \infty} \frac{1}{t} \int_{0}^{t} \int_{\mathbb{Y}} \ln \left(1+\gamma_{i}(s, u)\right) \tilde{N}(d s, d u)=0 \quad \text { a.s. }
$$

Moreover, it is easy to see that for any $t>s$,

$$
\int_{s}^{t} \sigma_{i}(r) d W(r)=\int_{0}^{t} \sigma_{i}(r) d W(r)-\int_{0}^{s} \sigma_{i}(r) d W(r)
$$

and

$$
\int_{s}^{t} \int_{\mathbb{Y}} \ln \left(1+\gamma_{i}(r, u)\right) \tilde{N}(d r, d u)=\int_{0}^{t} \int_{\mathbb{Y}} \ln \left(1+\gamma_{i}(r, u)\right) \tilde{N}(d r, d u)-\int_{0}^{s} \int_{\mathbb{Y}} \ln \left(1+\gamma_{i}(r, u)\right) \tilde{N}(d r, d u) .
$$

Consequently, for any $\epsilon>0$ we can deduce that there exists a constant $T>0$ such that

$$
\left|\int_{s}^{t} \sigma_{i}(r) d W(r)\right| \leq \epsilon(s+t) \quad \text { a.s. and }\left|\int_{s}^{t} \int_{\mathbb{Y}} \ln \left(1+\gamma_{i}(r, u)\right) \tilde{N}(d r, d u)\right| \leq \epsilon(s+t) \quad \text { a.s. }
$$

whenever $t>s \geq T$. Furthermore, by Lemma 4.2, together with (4.23), we have for $t \geq T$,

$$
\begin{aligned}
\frac{1}{Y_{i}(t)} \leq & \frac{1}{Y_{i}(T)} \exp \left(\int_{T}^{t}-\left[a_{i}(s)-\frac{1}{2} \sigma_{i}^{2}(s)+\int_{\mathbb{Y}}\left(\ln \left(1+\gamma_{i}(s, u)\right)-\gamma_{i}(s, u)\right) \lambda(d u)\right] d s+2 \epsilon(t+T)\right) \\
& +\int_{T}^{t} b_{i i}(s) \exp \left(-\int_{s}^{t}\left[a_{i}(r)-\frac{1}{2} \sigma_{i}^{2}(r)+\int_{\mathbb{Y}}\left(\ln \left(1+\gamma_{i}(r, u)\right)-\gamma_{i}(r, u)\right) \lambda(d u)\right] d r\right. \\
& +2 \epsilon(s+t)) d s, \quad \text { a.s. }
\end{aligned}
$$

This further gives that for any $t \geq T$,

$$
\begin{aligned}
e^{-4 \epsilon(t+T)} \frac{1}{Y_{i}(t)} \leq & \frac{1}{Y_{i}(T)} \exp \left(\int_{T}^{t}-\left[a_{i}(s)-\frac{1}{2} \sigma^{2}(s)+\int_{\mathbb{Y}}\left(\ln \left(1+\gamma_{i}(s, u)\right)-\gamma_{i}(s, u)\right) \lambda(d u)\right]\right) d s \\
& +\int_{T}^{t} b_{i i}(s) \exp \left(-\int_{s}^{t}\left[a_{i}(r)-\frac{1}{2} \sigma_{i}^{2}(r)+\int_{\mathbb{Y}}\left(\ln \left(1+\gamma_{i}(r, u)\right)-\gamma_{i}(r, u)\right) \lambda(d u)\right] d r\right. \\
& -2 \epsilon(t-s)-2 \epsilon T) d s, \quad \text { a.s. }
\end{aligned}
$$

Thus in view of (4.19) there exists a constant $K>0$ such that for any $t \geq T$,

$$
e^{-4 \epsilon(t+T)} \frac{1}{Y_{i}(t)} \leq K, \quad \text { a.s. }
$$


Hence for any $t \geq T$,

$$
\frac{1}{t} \ln \frac{1}{Y_{i}(t)} \leq 4 \epsilon\left(1+\frac{T}{t}\right)+\frac{1}{t} \ln K, \quad \text { a.s. }
$$

and the conclusion follows on supposing that $t \rightarrow \infty$ and from the arbitrariness of $\epsilon>0$.

\subsection{Further properties of n-dimensional competitive models}

We need the following lemma.

Lemma 4.5. Let the conditions of Theorem 4.4 hold. Assume further that for $i, j=1, \ldots, n$,

$$
R_{i j}:=\sup \left\{\frac{b_{i j}(t)}{b_{j j}(t)}, t \geq 0, i \neq j\right\}
$$

satisfy

$$
R_{i}(t)-\sum_{i \neq j} R_{i j} R_{j}(t)>0, \quad t \geq 0 .
$$

Then

$$
\liminf _{t \rightarrow \infty} \frac{\ln Z_{i}(t)}{t} \geq 0, \quad \text { a.s. }
$$

where $Z_{i}(t), i=1, \ldots, n$, are solutions of $(2.5)$.

Remark 4.1. For $i, j=1, \ldots, n$ and $t \geq 0$, if $b_{i j}(t)$ takes finite-number values, then condition (4.24) must hold.

Proof. It is sufficient to show that $\limsup _{t \rightarrow \infty} \frac{1}{t} \ln \frac{1}{Z_{i}(t)} \leq 0$. Note from Lemma 4.2 that for any $t>s \geq 0$,

$$
\begin{aligned}
\frac{1}{Z_{i}(t)}= & \frac{1}{Z_{i}(s)} \exp \left(\int_{s}^{t}-\left[a_{i}(r)-\sum_{i \neq j} b_{i j}(r) Y_{j}(r)-\frac{1}{2} \sigma_{i}^{2}(r)+\int_{\mathbb{Y}}\left(\ln \left(1+\gamma_{i}(r, u)\right)-\gamma_{i}(r, u)\right) \lambda(d u)\right] d r\right. \\
& \left.-\int_{s}^{t} \sigma_{i}(s) d W(s)-\int_{s}^{t} \int_{\mathbb{Y}} \ln \left(1+\gamma_{i}(s, u)\right) \tilde{N}(d s, d u)\right) \\
& +\int_{s}^{t} b_{i i}(r) \exp \left(-\int_{r}^{t}\left[a_{i}(\tau)-\sum_{i \neq j} b_{i j}(\tau) Y_{j}(\tau)-\frac{1}{2} \sigma_{i}^{2}(\tau)\right.\right. \\
& \left.+\int_{\mathbb{Y}}\left(\ln \left(1+\gamma_{i}(\tau, u)\right)-\gamma_{i}(\tau, u)\right) \lambda(d u)\right] d \tau \\
& \left.-\int_{r}^{t} \sigma_{i}(\tau) d W(\tau)-\int_{r}^{t} \int_{\mathbb{Y}} \ln \left(1+\gamma_{i}(\tau, u)\right) \tilde{N}(d \tau, d u)\right) d r .
\end{aligned}
$$

Applying the Itô formula, for any $t>s \geq 0$,

$$
\begin{aligned}
\int_{s}^{t} b_{i i}(r) Y_{i}(r) d r= & \ln Y_{i}(s)-\ln Y_{i}(t)+\int_{s}^{t}\left[a_{i}(r)-\frac{1}{2} \sigma_{i}^{2}(r)+\int_{\mathbb{Y}}\left(\ln \left(1+\gamma_{i}(r, u)\right)-\gamma_{i}(r, u)\right) \lambda(d u)\right] d s \\
& +\int_{s}^{t} \sigma_{i}(r) d W(r)+\int_{s}^{t} \int_{\mathbb{Y}} \ln \left(1+\gamma_{i}(r, u)\right) \tilde{N}(d r, d u) .
\end{aligned}
$$

This, together with Theorem 4.4 and (4.23), yields that for any $\epsilon>0$ there exists $\bar{T}>0$ such that

$$
\int_{s}^{t} b_{i i}(r) Y_{i}(r) d r \leq \int_{s}^{t}\left[a_{i}(r)-\frac{1}{2} \sigma_{i}^{2}(r)+\int_{\mathbb{Y}}\left(\ln \left(1+\gamma_{i}(r, u)\right)-\gamma_{i}(r, u)\right) \lambda(d u)\right] d s+3 \epsilon(s+t)
$$

whenever $t \geq s \geq \bar{T}$. Moreover taking into account (4.28) and (4.29), we have for $t>s \geq \bar{T}$,

$$
\begin{aligned}
\int_{s}^{t} b_{i j}(r) Y_{j}(r) d r & =\int_{s}^{t} \frac{b_{i j}(r)}{b_{j j}(r)} b_{j j}(r) Y_{j}(r) d r \\
& \leq R_{i j} \int_{s}^{t} b_{j j}(r) Y_{j}(r) d r \\
& \leq 3 \epsilon(s+t) R_{i j}+\int_{s}^{t} R_{i j}\left[a_{i}(r)-\frac{1}{2} \sigma_{i}^{2}(r)+\int_{\mathbb{Y}}\left(\ln \left(1+\gamma_{i}(r, u)\right)-\gamma_{i}(r, u)\right) \lambda(d u)\right] d s .
\end{aligned}
$$


Putting this into (4.27) leads to

$$
\begin{aligned}
\frac{1}{Z_{i}(t)}= & \frac{1}{Z_{i}(s)} \exp \left(-\int_{s}^{t}\left[R_{i}(r)-\sum_{i \neq j} R_{i j} R_{j}(r)\right] d r+\epsilon(s+t)\left(3 \sum_{i \neq j} R_{i j}+2\right)\right) \\
& +\int_{s}^{t} b_{i i}(r) \exp \left(-\int_{r}^{t}\left[R_{i}(\tau)-\sum_{i \neq j} R_{i j} R_{j}(\tau)\right] d \tau+\epsilon(r+t)\left(3 \sum_{i \neq j} R_{i j}+2\right)\right) d r,
\end{aligned}
$$

which, in addition to (4.25), implies

$$
\frac{1}{Z_{i}(t)}=\frac{1}{Z_{i}(s)} \exp \left(\epsilon(s+t)\left(3 \sum_{i \neq j} R_{i j}+2\right)\right)+\int_{s}^{t} b_{i i}(r) \exp \left(\epsilon(r+t)\left(3 \sum_{i \neq j} R_{i j}+2\right)\right) d r .
$$

By arguments similar to those of Theorem 4.4, we can deduce that there exists $K>0$ such that for $t>s \geq \bar{T}$,

$$
\exp \left(-2 \epsilon(s+t)\left(3 \sum_{i \neq j} R_{i j}+2\right)\right) \frac{1}{Z_{i}(t)} \leq K
$$

and the conclusion follows.

Now a combination of Theorem 4.4 and Lemma 4.5 gives the following theorem.

Theorem 4.5. Under the conditions of Lemma 4.5, for each $i=1, \ldots, n$,

$$
\lim _{t \rightarrow \infty} \frac{\ln X_{i}(t)}{t}=0, \quad \text { a.s. }
$$

Another important property of a population dynamics is the extinction, which means that every species will become extinct. The most natural analogue for the stochastic population dynamics (1.5) is that every species will become extinct with probability 1 . To be precise, let us give the definition.

Definition 4.2. Stochastic population dynamics (1.5) is said to be extinct with probability 1 if, for every initial datum $x_{0} \in \mathbb{R}_{+}^{n}$, the solution $X_{i}(t), t \geq 0$, has the property

$$
\lim _{t \rightarrow \infty} X_{i}(t) \rightarrow 0 \text { a.s.. }
$$

Theorem 4.6. Let assumption (A) and (4.20) hold. Assume further that

$$
\eta_{i}:=\limsup _{t \rightarrow \infty} \frac{1}{t} \int_{0}^{t} \beta_{i}(s) d s<0,
$$

where, for $t \geq 0$ and $i=1, \ldots, n$,

$$
\beta_{i}(t):=a_{i}(t)-\frac{1}{2} \sigma_{i}^{2}(t)-\int_{\mathbb{Y}}\left(\gamma_{i}(t, u)-\ln \left(1+\gamma_{i}(t, u)\right)\right) \lambda(d u) .
$$

Then stochastic population dynamics (1.5) is extinct a.s.

Proof. Recalling by the comparison theorem that, for any $t \geq 0$ and $i=1, \ldots, n$,

$$
X_{i}(t) \leq Y_{i}(t)
$$

we only need to verify that $\lim \sup _{t \rightarrow \infty} Y_{i}(t)=0$ a.s., due to

$$
0 \leq \liminf _{t \rightarrow \infty} X_{i}(t) \leq \limsup _{t \rightarrow \infty} X_{i}(t) \leq \limsup _{t \rightarrow \infty} Y_{i}(t) .
$$

Since $b_{i}(t) \geq 0$, by (4.7) it is easy to observe that

$$
\begin{aligned}
Y_{i}(t) & \leq X_{i}(0) \exp \left(\int_{0}^{t} \beta_{i}(s) d s+\int_{0}^{t} \sigma_{i}(s) d W(s)+\int_{0}^{t} \int_{\mathbb{Y}} \ln \left(1+\gamma_{i}(s, u)\right) \tilde{N}(d s, d u)\right) \\
& =X_{i}(0) \exp \left(t\left(\frac{1}{t} \int_{0}^{t} \beta_{i}(s) d s+\frac{1}{t} \int_{0}^{t} \sigma_{i}(s) d W(s)+\frac{1}{t} \int_{0}^{t} \int_{\mathbb{Y}} \ln \left(1+\gamma_{i}(s, u)\right) \tilde{N}(d s, d u)\right)\right) .
\end{aligned}
$$

Thanks to $\eta_{i}<0$, in addition to (4.22), we deduce that $\lim \sup _{t \rightarrow \infty} Y_{i}(t)=0$ a.s. and the conclusion follows.

Remark 4.2. In Theorem 4.3, we know that our one-dimensional model has a unique invariant measure under some conditions; however we cannot obtain the same result for the $n$-dimensional model $(n \geq 2)$. 


\section{Conclusions and further remarks}

In this paper, we discuss competitive Lotka-Volterra population dynamics with jumps. We show that the model admits a unique global positive solution, and investigate the uniformly finite $p$ th moment with $p>0$, stochastic ultimate boundedness, invariant measure and long-term behaviours of solutions. Moreover, using a variation-of-constants formula for a class of SDEs with jumps, we provide an explicit solution for the model, and investigate precisely the sample Lyapunov exponent for each component and the extinction of our $n$-dimensional model.

As we mentioned in the introduction section, random perturbation of interspecies or intraspecies interactions by white noise is one of the ways to perturb population dynamics. In [9], Mao et al. investigated the stochastic $n$-dimensional Lotka-Volterra systems

$$
d X(t)=\operatorname{diag}\left(X_{1}(t), \ldots, X_{n}(t)\right)[(a+B X(t)) d t+\sigma X(t) d W(t)],
$$

where $a=\left(a_{1}, \ldots, a_{n}\right)^{T}, B=\left(b_{i j}\right)_{n \times n}, \sigma=\left(\sigma_{i j}\right)_{n \times n}$. It would be interesting to know what would happen if the stochastic Lotka-Volterra systems (5.1) were further perturbed by jump diffusions, namely

$$
d X(t)=\operatorname{diag}\left(X_{1}\left(t^{-}\right), \ldots, X_{n}\left(t^{-}\right)\right)\left[\left(a+B X\left(t^{-}\right)\right) d t+\sigma X(t) d W(t)+\int_{\mathbb{Y}} \gamma\left(X\left(t^{-}\right), u\right) \tilde{N}(d t, d u)\right]
$$

where $\gamma=\left(\gamma_{1}, \ldots, \gamma_{n}\right)^{T}$. On the other hand, the hybrid systems driven by continuous-time Markov chains have been used to model many practical systems, where there may be abrupt changes in the structure and parameters caused by phenomena such as environmental disturbances [11]. As mentioned in Zhu and Yin [15,16], interspecies and intraspecies interactions are often subject to environmental noise, and the qualitative changes cannot be described using the traditional (deterministic or stochastic) Lotka-Volterra models. For example, interspecies and intraspecies interactions often vary according to changes in nutrition and/or food resources. We use the continuous-time Markov chain $r(t)$ with a finite state space $\mathcal{M}=\{1, \ldots, m\}$ to model these abrupt changes, and need to deal with the stochastic hybrid population dynamics with jumps

$$
\begin{aligned}
d X(t)= & \operatorname{diag}\left(X_{1}\left(t^{-}\right), \ldots, X_{n}\left(t^{-}\right)\right)\left[\left(a(r(t))+B(r(t)) X\left(t^{-}\right)\right) d t+\sigma(r(t)) X\left(t^{-}\right) d W(t)\right. \\
& \left.+\int_{\mathbb{Y}} \gamma\left(X\left(t^{-}\right), r(t), u\right) \tilde{N}(d t, d u)\right] .
\end{aligned}
$$

We will report our findings in our following papers.

\section{References}

[1] K. Gopalsamy, Stability and Oscillations in Delay Differential Equations of Population Dynamics, Kluwer Academic, Dordrecht, 1992.

[2] Y. Kuang, Delay Differential Equations with Applications in Population Dynamics, Academic Press, Boston, 1993.

[3] X. Li, C. Tang, X. Ji, The criteria for globally stable equilibrium in $n$-dimensional Lotka-Volterra systems, J. Math. Anal. Appl. 240 (1999) 600-606.

[4] Y. Takeuchi, N. Adachi, The existence of globally stable equilibria of ecosystems of the generalized Volterra type, J. Math. Biol. 10 (1980) 401-415.

[5] Y. Takeuchi, N. Adachi, The stability of generalized Volterra equations, J. Math. Anal. Appl. 62 (1978) $453-473$.

[6] D. Xiao, W. Li, Limit cycles for the competitive three dimensional Lotka-Volterra system, J. Differential Equations 164 (2000) 1-15.

[7] T. Gard, Persistence in stochastic food web models, Bull. Math. Biol. 46 (1984) 357-370.

[8] T. Gard, Stability for multispecies population models in random environments, Nonlinear Anal. 10 (1986) $1411-1419$.

[9] X. Mao, G. Marion, E. Renshaw, Environmental noise suppresses explosion in population dynamics, Stochastic Process. Appl. 97 (2002) $95-110$.

[10] X. Mao, C. Yuan, J. Zou, Stochastic differential delay equations of population dynamics, J. Math. Anal. Appl. 304 (2005) $296-320$.

[11] X. Mao, C. Yuan, Stochastic Differential Equations with Markovian Switching, Imperial College Press, 2006.

[12] G. Hu, K. Wang, On stochastic logistic equation with Markovian switching and white noise, Osaka J. Math. (2010) (preprint).

[13] D. Jiang, N. Shi, A note on nonautonomous logistic equation with random perturbation, J. Math. Anal. Appl. 303 (2005) $164-172$.

[14] M. Liu, K. Wang, Persistence and extinction in stochastic non-autonomous logistic systems, J. Math. Anal. Appl. 375 (2011) $443-457$.

[15] C. Zhu, G. Yin, On hybrid competitive Lotka-Volterra ecosystems, Nonlinear Anal. 71 (2009) e1370-e1379.

[16] C. Zhu, G. Yin, On competitive Lotka-Volterra model in random environments, J. Math. Anal. Appl. 357 (2009) 154-170.

[17] D. Roubik, Experimental community studies: time-series tests of competition between African and neotropical bees, Ecology 64 (1983) 971-978.

[18] J. Roughgarden, Theory of Population Genetics and Evolutionary Ecology: An Introduction, Macmillan, New York, 1979.

[19] S. Peng, X. Zhu, Necessary and sufficient condition for comparison theorem of 1-dimensional stochastic differential equations, Stochastic Process. Appl. 116 (2006) 370-380.

[20] D. Prato, J. Zabczyk, Ergodicity for Infinite Dimensional Systems, Cambridge University Press, 1996.

[21] R. Lipster, A strong law of large numbers for local martingales, Stochastics 3 (1980) 217-228.

[22] H. Kunita, Itô's stochastic calculus: its surprising power for applications, Stochastic Process. Appl. 120 (2010) $622-652$.

[23] B. Øksendal, A. Sulem, Applied Stochastic Control of Jump Diffusions, 2nd ed., Springer, Berlin, 2007.

[24] D. Applebaum, Lévy Processes and Stochastics Calculus, 2nd ed., Cambridge University Press, 2009. 